\title{
Heterologous Protection to COVID-19 with BCG Vaccine: Deciphering the Reality Using Meta-Analysis Approach
}

\author{
Anurag Singh', Lakshya Gupta², Vandana Gupta ${ }^{1 *}$
}

'Department of Microbiology, Ram Lal Anand College, University of Delhi, Benito Juarez Road, New Delhi 110021, India ${ }^{2}$ Department of Computer Science and Engineering, Indian Institute of Technology, Varanasi, Uttar Pradesh 221005, India.

Article Info

\section{Article Notes}

Received: October 07, 2020

Accepted: December 11, 2020

\section{*Correspondence:}

*Dr. Vandana Gupta, Department of Microbiology, Ram Lal Anand College, University of Delhi, Benito Juarez Road, New Delhi 110021, India; Email: vandanagupta72@rediffmail.com.

(c) 2020 Gupta V. This article is distributed under the terms of the Creative Commons Attribution 4.0 International License.

\section{Keywords}

BCG vaccination

COVID-19

Heterologous protection

SARS-CoV-2

Innate Immunity

\section{ABSTRACT}

The coronavirus disease (COVID-19) emerged in China in December 2019 and has since spread to over 188 countries affecting millions of individuals. Several reports in favour or against the heterologous protection conferred by the BCG vaccine against COVID-19 came up in the initial days of the pandemic and continue to do so. In this study, we compared the three worst-affected nations: The USA, India, and Brazil, their current pandemic scenario, and their respective national BCG immunization policies. USA recommends BCG vaccine only to a specific group of people and never had a national immunization scheme in place. Meanwhile, India introduced a nationwide scheme as early as 1948 and continues to endorse BCG immunization at birth. Brazil used the oral route to administer the BCG vaccine till 1976, and then shifted to intradermal injection. The correlation coefficient for the total number of cases, cases per million, the total number of deaths, deaths per million and case fatality rate ranges between any two countries under study. This indicates a very strong positive correlation in the various epidemiological parameters between countries with no national immunization scheme (USA) and countries with stringent national policies on BCG vaccination. The strongest correlation exists between the USA and Brazil followed by Brazil and India which is very closely followed by the USA and India. We found no consistent evidence to infer in favour of the hypothesis that BCG provides any non-specific protection against COVID-19.

\section{Abbreviations}

BCG: Bacillus Calmette-Guérin

COVID-19: Coronavirus Disease 2019

DPT: Diphtheria, Pertussis, and Tetanus

HPV: Human Papillomavirus

HSV: Herpes Simplex Virus

ICTV: International Committee on Taxonomy of Viruses

OPV: Oral Poliovirus Vaccine

RSV: Respiratory Syncytial Virus

SARS-CoV-2: Severe Acute Respiratory Syndrome Coronavirus 2

TB: Tuberculosis

WHO: World Health Organization

\section{Introduction}

It all started back in December 2019 when the case(s) of pneumonia of unknown etiology came up in Wuhan, China, and spread across the world in over 188 countries within months. 
Investigations and whole-genome sequencing indicated the origin of a new coronavirus that has skipped from its natural reservoir "bat" intermediate host "pangolin" 2 that is sold illegally in Chinese markets. This suggested a zoonotic origin like many other newly emerged viruses including Nipah, SARS-CoV, MERS-CoV, Ebola, Marburg, etc. This contagious virus was initially named by WHO as 2019-nCoV (2019novel Coronavirus), and subsequently the International Committee on Taxonomy of Viruses (ICTV) renamed it as SARS-CoV-2 (Severe Acute Respiratory Syndrome Coronavirus 2) ${ }^{3}$. SARS-CoV-2 gets rapidly transmitted to naïve population, from the infected patients essentially via contaminated droplets and fomites ${ }^{4,5}$. However, cases of shedding of viral RNA in feces ${ }^{6}$ have also been documented.

As of September 17, 2020, there have been over 29,674,488 laboratory-confirmed cases of coronavirus disease (COVID-19) worldwide with more than 937,204 fatalities (https://coronavirus.jhu.edu). Patients infected with SARS-CoV-2 present symptoms like fever, sore throat, fatigue, phlegm production, loss of smell or taste, headache, etc. among others which may appear after 2-14 days postexposure ${ }^{7}$, with a mean incubation period of 5.2 days $^{8}$. In the initial days of the outbreak, when the world was still struggling to establish uniform treatment protocols, various reports of the effectiveness of BCG vaccination to protect against COVID-19, or at least bridge the treatment gap came up. It was reported that "the countries which didn't have a universal BCG vaccination policy suffered more severely than those with universal and long-standing policies" in a study by the scientists at the New York Institute of Technology ${ }^{9}$. It was further indicated that substantial bearing on mortality and morbidity due to COVID-19 might be determined by the BGC vaccination. The previously mentioned study also contested that an early establishment of a uniform BCG vaccination policy by some countries would see a significant difference in mortality rate, which is in line with the globally recognized tendency of the elderly being more vulnerable to COVID-19 related mortalities. Similar conclusions are very recently reported by researchers from St Petersburg University, Russia ${ }^{10}$.

Albert Calmette and Camille Guérin prepared the BCG vaccine by attenuating a live isolate of Mycobacterium bovis that elicits a strong immune response and is known to protect children from disseminated forms of tuberculosis (TB) and can also confer limited protection in adults against pulmonary tuberculosis ${ }^{11}$. It is also known to bestow 'nonspecific' or 'heterologous' protection against many unrelated pathogens which includes viruses such as RSV, HPV, and $\mathrm{HSV}^{11,12}$. A randomized control trial also reported that BCG vaccination significantly lowered viremia when challenged with yellow fever virus vaccine strain ${ }^{13}$. Yet another trial found a more pronounced antibody response against influenza A (H1N1) vaccine strain when it is administered 14 days after BCG vaccination ${ }^{14}$. Among other examples, a reduction in neonatal mortality, primarily due to fewer cases of respiratory infection, neonatal sepsis, and fever were linked to BCG vaccination in a randomized trial among lowbirth-weight children in Guinea-Bissau ${ }^{15}$. Moreover, in an area with high mortality in West Africa, the scar from BCG vaccination and a positive tuberculin reaction were linked with better survival in early childhood ${ }^{16}$.

Through this article, we intend to shed light on the current scenario of the pandemic in the three worstaffected countries: The United States of America, India, and Brazil; their national BCG vaccination policy, if any; and if past vaccination with BCG has affected the number or the outcome of SARS-CoV-2 infections in the patients.

\section{Methodology}

We did a systematic literature search to track the past developments related to the national BCG immunization scheme of the USA, India, and Brazil, and its current implications in SARS-CoV-2 outbreak by recollecting information from online databases (primarily PubMed), by using keywords like 'BCG', 'BCG vaccination policy' and 'BCG and SARS-CoV-2'. Other essential information regarding vaccination was filtered from $\mathrm{WHO}^{17}$ (https://apps. who.int/gho/data/node.main.A830?lang=en) and 'BCG World Atlas' ${ }^{18}$ (http://www.bcgatlas.org/index.php). The COVID-19 patient data used in this analysis was obtained from 'Our World in Data' ${ }^{19}$ (https://ourworldindata.org/ coronavirus) (as of 17th Sep 2020). Moreover, information regarding clinical trials was retrieved from ClinicalTrials. gov (http://clinicaltrials.gov/) registry and Clinical Trials Registry- India (http://ctri.nic.in/). All the country wise data concerning the total number of cases and fatality is included irrespective of age, comorbidities, gender, ethnic groups, etc. The measure of dependency between the relative movements of two variables (no. of COVID-19 cases and mortalities in two different countries) is statistically quantified in this study using the Pearson correlation. The correlation coefficient is the ratio of covariance between the two variables and the product of the variance of the individual variables. Values of the correlation coefficient lie between -1.0 and 1.0. A value of -1.0 depicts a perfect negative correlation; in contrast, a value of 1.0 indicates a perfect positive correlation. A zero value exhibits a lack of any co-relation. A statistical measure of correlation coefficient was applied in a python environment with the script provided in the supplementary information.

\section{Results and Discussion}

\section{Timeline of BCG Vaccination in India, Brazil, and the USA and the current population coverage}

Data on the introduction and the inclusion of BCG 


\section{BCG Immunization coverage estimates}

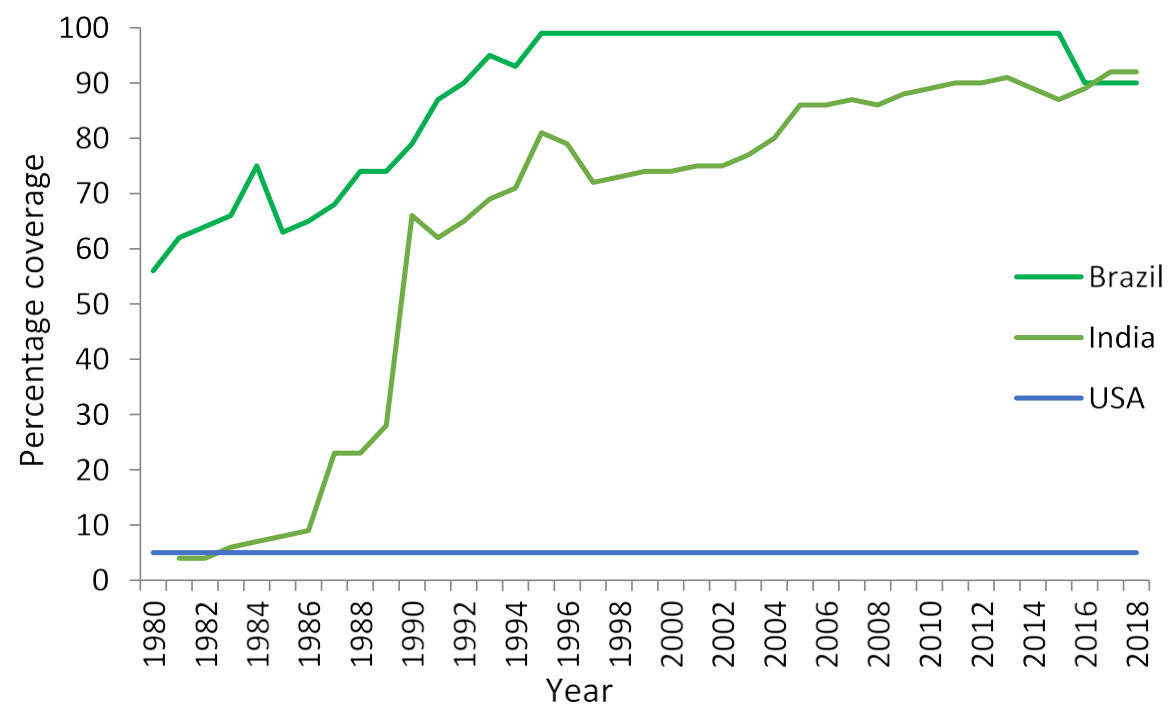

Figure 1: BCG immunization coverage estimates of India and Brazil. The USA never had a nationwide BCG immunization scheme (here the coverage in the USA have been plotted between $0-5 \%$ population for the sake of comparison)

[Reference: $\mathrm{WHO}^{17}$ ]

vaccination in Government compulsory vaccination policy was compiled for the three countries under the current study (Figure 1) to deduce the effective population coverage.

India: India introduced the BCG vaccine in 1948 with assistance from the International Tuberculosis Campaign (ITC). In 1951, the Government of India endorsed expanding the campaign across the country. It was initially aimed to immunize individuals in the 1-25 year age group ${ }^{20}$ but was later revised to immunize babies within a year after birth, based on the epidemiological studies suggesting that BCG could protect from childhood forms of $\mathrm{TB}^{21}$. The Expanded Programme of Immunization was then introduced in 1978 to cover $80 \%$ of infants ${ }^{22}$, however, it remained confined to urban areas. On November 19, 1985, a Universal Immunization Programme (UIP) was launched to increase the coverage in all the districts by 1990 . All these efforts lead to the effective BCG vaccine coverage of approximately $90 \%$ by the year 2016 (Figure 1).

Brazil: BCG vaccine sample distributed by Pasteur Institute arrived in Brazil as early as 1925, however, it started to gain acceptance slowly and in the year 1944 alone, about 19000 babies were vaccinated; and the supply chain to different states was developed. From 1977 onwards a $0.05 \mathrm{ml}$ intradermic dose for new-borns followed by revaccination at school-age was recommended. This schedule was modified in 1982 and a single dose of $0.1 \mathrm{ml}$ was recommended for neonates [reviewed in ${ }^{23}$. The population coverage in Brazil reached its peak $(100 \%)$ as early as 1994 and remained so for almost 20 years, with a decline of approximately $10 \%$ seen in recent years (Figure 1).
Table 1: SARS-CoV-2 infection/ mortality data (as of 17th Sep 2020) in the three countries under study [Reference: European Centre for Disease Prevention and Control (ECDC) and Our World in Data ${ }^{19}$ ]

\begin{tabular}{|l|l|l|l|l|l|}
\hline Country & $\begin{array}{l}\text { Total cases } \\
\text { (no.) }\end{array}$ & $\begin{array}{l}\text { Total Deaths } \\
\text { (no.) }\end{array}$ & $\begin{array}{l}\text { Caseload } \\
\text { / million }\end{array}$ & $\begin{array}{l}\text { Deaths/ } \\
\text { million }\end{array}$ & $\begin{array}{l}\text { Case Fatality } \\
\text { rate (\%) }\end{array}$ \\
\hline USA & $6,616,458$ & 196,436 & 19958.43 & 591.95 & 2.96 \\
\hline Brazil & $4,382,263$ & 133,116 & 20616.65 & 626.27 & 3.03 \\
\hline India & $5,020,359$ & 82,066 & 3637.93 & 59.468 & 1.63 \\
\hline
\end{tabular}

According to a WHO estimate (Figure 1), by 2018, India and Brazil had an excellent countrywide BCG immunization coverage ( $92 \%$ and $90 \%$ respectively $)^{17}$.

USA: In the United States of America, BCG vaccination is considered only for specific groups and is not used widely. Since the USA never had a universal BCG vaccination policy and no data is available for population coverage, we have plotted at $5 \%$ population coverage for the sake of comparison (Figure1).

\section{Current scenario of SARS-CoV-2 infections and Case fatality rates (CFR) in the three countries under study}

According to Johns Hopkins University and Medicine Coronavirus Resource Center (https://coronavirus.jhu. edu), as of September 17, 2020, the data for the total number of infections and deaths, per million caseload and deaths and the case fatality rate is given in table 1 . The per million population data ${ }^{19}$ for caseload and mortality could be a better indicator for comparison. The USA and Brazil had almost equal caseload/million of 19958.43 and 20616.65 respectively. The mortality rate in Brazil was slightly higher than in the USA. Both the cases and mortality per million 
people in India were comparatively fairly low which may be attributed to the stage of pandemic and also to the strict prolonged lockdown in the country. Till the cut-off time of this study, the daily new cases were on the increasing trend post different stages of unlocking in India which didn't
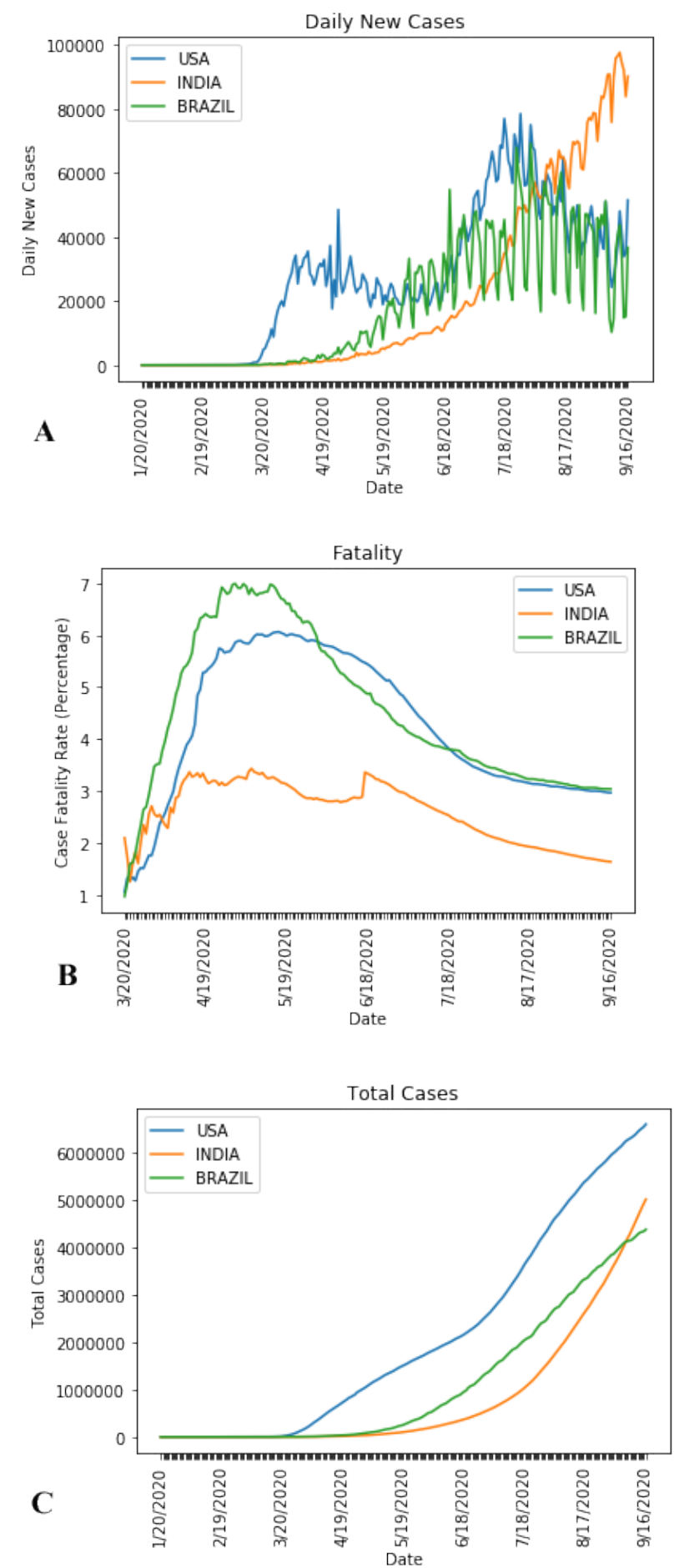

reach its plateau, whereas, the USA and Brazil were showing a decreasing trend in terms of daily new cases (Figure $2 a$ ). Case fatality rates were on a decline in these three countries as was also observed for many other countries in the world (Figure $2 b$ ), which could potentially be associated with
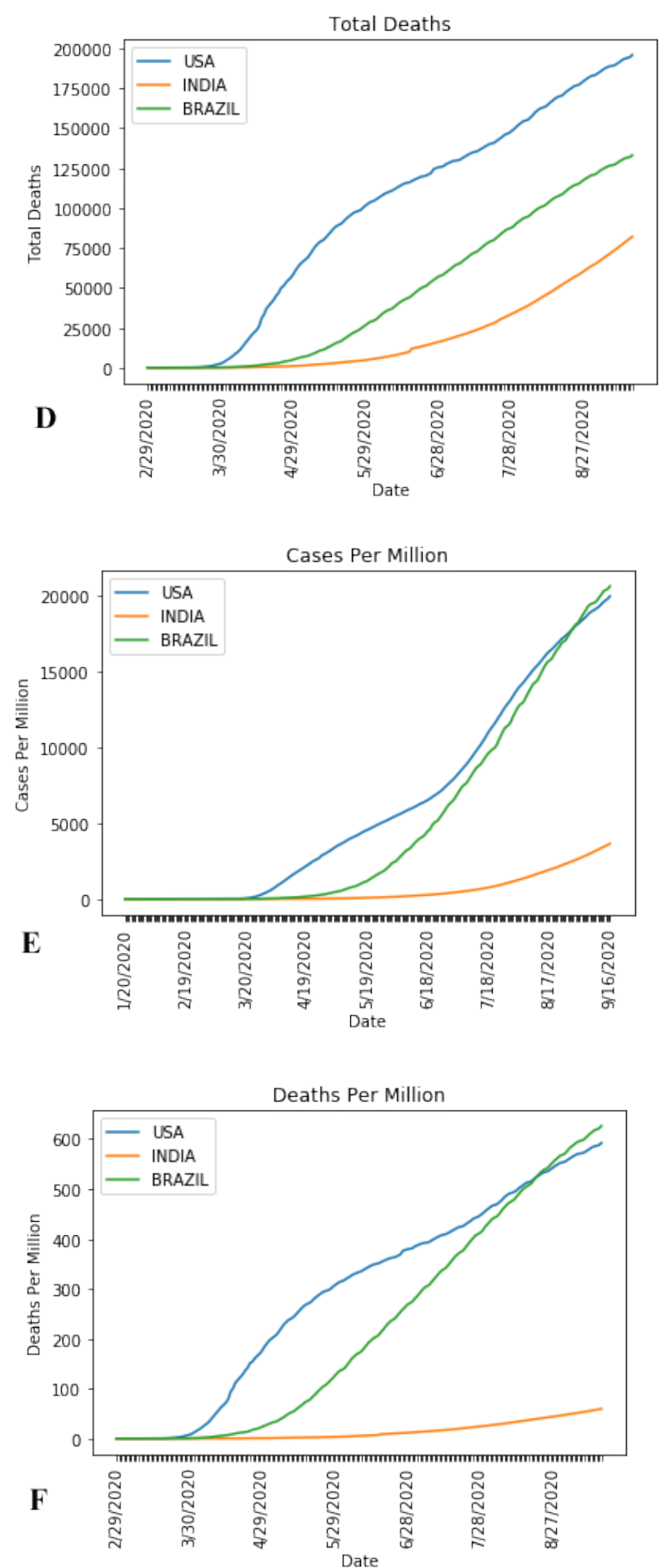

Figure 2: Comparison of COVID-19 patient data between the USA, India, and Brazil: (a) Daily new cases showing declining trends in the USA and Brazil, whereas, trends in India are on the rise; (b) Case fatality rate depicting similar trends in all three countries; (c) Total confirmed cases; (d) Total confirmed deaths; (e) Total confirmed cases per million people; and (f) Total confirmed deaths per million people. [Reference: European Centre for Disease Prevention and Control (ECDC) and Our World in Data ${ }^{19}$ ] 
Table 2: Correlation coefficient between countries under study.

\begin{tabular}{|l|c|c|c|}
\hline \multirow{2}{*}{ Parameters } & \multicolumn{3}{|c|}{ Correlation Coefficient } \\
\cline { 2 - 4 } & USA-Brazil & Brazil-India & India-USA \\
\hline Cases/Cases per million & 0.98 & 0.96 & 0.93 \\
\hline Deaths/ Deaths per million & 0.94 & 0.96 & 0.85 \\
\hline Case fatality rate & 0.90 & 0.86 & 0.81 \\
\hline
\end{tabular}

Table 3: On-going clinical trials to ascertain the correlation between BCG vaccine and COVID-19.

[Reference: ClinicalTrials.gov (https://clinicaltrials.gov/) registry and Clinical Trials Registry- India (http://ctri.nic.in/).]

\begin{tabular}{|l|l|l|l|l|l|l|}
\hline Identifier & Model & $\begin{array}{l}\text { Estimated } \\
\text { Enrolment }\end{array}$ & Recruitment Status & Phase & Location & Last Update \\
\hline NCT04327206 & RCT & 10078 & Recruiting & Phase 3 & $\begin{array}{l}\text { Australia, Netherlands, } \\
\text { Spain }\end{array}$ & August 20, 2020 \\
\hline NCT04328441 & RCT & 1500 & Active, not recruiting & Phase 3 & Netherlands & August 19, 2020 \\
\hline CTRI/2020/04/024749 & RCT & 5946 & $\begin{array}{l}\text { Closed to Recruitment } \\
\text { of Participants }\end{array}$ & Phase 3 & India & July 22, 2020 \\
\hline CTRI/2020/04/024833 & RCT & 1826 & Not Yet Recruiting & N/A & India & June 01, 2020 \\
\hline CTRI/2020/06/025854 & $\begin{array}{l}\text { Non-randomized, Active } \\
\text { Controlled Trial }\end{array}$ & 1450 & Not Yet Recruiting & N/A & India & June 08, 2020 \\
\hline CTRI/2020/07/026668 & RCT & 800 & Not Yet Recruiting & N/A & India & July 17, 2020 \\
\hline CTRI/2020/09/027684 & RCT & 400 & Open to Recruitment & N/A & India & $\begin{array}{l}\text { September 29, } \\
2020\end{array}$ \\
\hline
\end{tabular}

Abbreviation(s): RCT (Randomized Control Trial)

a more standardised treatment protocol. The cases and mortality per million for Brazil were even higher than that of the USA, clearly suggesting no consistent effect on infection counts or fatalities between countries having a national vaccination policy over countries that never had a nationwide BCG immunization scheme. A comparison of the pattern of disease progression in the three countries in terms of the total number of cases and death (Figure $2 c$ and $2 d$ ), and caseload and deaths per million (figure $2 e$ and 2f) reported each day, depict a linear correlation. This is further confirmed by determining the correlation coefficient, which shows a very strong positive correlation between total cases and case fatality rates between any two of the three countries (Table 2). It is important to note here that the data in the graph represents the data till 17th Sep 2020. The caseload at present, however, is again shooting up, suggesting a fresh wave of infection in most of the countries.

As the Live-attenuated vaccine like BCG and measles are known to induce heterologous protection ${ }^{24}$ has been reported to provide 'non-specific' or 'heterologous' protection to many unrelated pathogens including viruses such as RSV, HPV, HSV, yellow fever and influenza $\mathrm{A}^{11-14}$, it seemed logical to infer the conceivable heterologous protection it seemed to have provided to SARS-CoV-2. Possibly through the suggested mechanism of this offtarget protection that parallels to the maturation of Th1 response, 'trained' monocytes and natural killer (NK) cells which enables strong and rapid response to stimulation by heterologous antigens. Primed monocytes express more surface markers and are involved in the increased production of cytokines. This is regulated by epigenetic changes (histone modification), and metabolic shift (oxidative phosphorylation to glycolysis) ${ }^{12}$. On the contrary, in this study, we could demonstrate that there is no correlation between BCG coverage and heterologous protection to SARS-CoV-2. A similar rate of COVID-19 positive test results between vaccinated and non-vaccinated individuals has been reported by other worker ${ }^{25,26}$. A more recent study by Hensel and co-workers ${ }^{27}$ suggested SARS-CoV-2 testing as an important bias in determining a correlation between BCG vaccination and COVID-19 spread. In countries with high testing rates $(>=10$ per thousand inhabitants), the authors failed to determine a significant difference between COVID-19 spread rates among countries with different (or no) BCG vaccination policies. This study also cautioned against differences in cases at the 'subnational level' being extrapolated to the entire country, and the prevalence of different strains of the virus as an important set of confounders. Randomized control studies could provide stronger evidence to this new prospect of BCG vaccination and multiple studies are already underway (Table 3).

\section{Conclusion}

The SARS-CoV- 2 infection and mortality data of the three worst-affected nations provides a unique combination for comparatively analysing whether BCG is exerting heterologous protection against SARS-CoV-2 infection. It has to be acknowledged that any statistical evidence for or against the use of the BCG vaccine in the current scenario is bound by potential confounders owing to a dynamic 
pandemic situation. Criteria such as demography, the genetic makeup of a population, stage of the pandemic infection control measures adopted, number of tests done, existing co-morbid conditions, healthcare infrastructure, governmental preparedness, data transparency, etc. can impose significant bias in studies ${ }^{28}$. It is also not ascertained whether the elderly population who had been vaccinated several years ago would still maintain a well 'trained immunity' ${ }^{28}$. In the absence of any firm evidence, the WHO currently does not recommend using the BCG vaccine for protection from COVID-1929. In line with earlier studies ${ }^{25-27}$, our study strongly disproves the hypothesis that "BCG vaccine confers any non-specific protection to COVID-19". Though multiple underway randomized trials, when completed could better reflect on this facet of BCG vaccination.

\section{Declaration}

This study has not been funded by any agency.

\section{Acknowledgments}

We would like to acknowledge Dr. Rakesh K Gupta for his continuous motivation and support.

\section{Conflicts of interest}

None

\section{References}

1. Zhou P, Yang X, Wang X, et al. A pneumonia outbreak associated with a new coronavirus of probable bat origin. Nature. 2020; 579(March). doi:10.1038/s41586-020-2012-7

2. Zhang C, Zheng W, Huang X, et al. Protein Structure and Sequence Reanalysis of 2019-nCoV Genome Refutes Snakes as Its Intermediate Host and the Unique Similarity between Its Spike Protein Insertions and HIV-1. J Proteome Res. 2020; 19(4): 1351-1360. doi:10.1021/acs. jproteome.0c00129

3. Naming the coronavirus disease (COVID-19) and the virus that causes it. Accessed September 16, 2020. https://www.who.int/emergencies/ diseases/novel-coronavirus-2019/technical-guidance/naming-thecoronavirus-disease-(covid-2019)-and-the-virus-that-causes-it

4. Report of the WHO-China Joint Mission on Coronavirus Disease 2019 (COVID-19 ). 2020; 2019(February): 16-24.

5. Ying Chia P, Kelli Coleman K, Kim Tan Y, et al. Detection of air and surface contamination by SARS-CoV-2 in hospital rooms of infected patients. Nat Commun. 2020; 11(1): 2800. doi:10.1038/s41467-020-16670-2

6. Wu Y, Guo C, Tang $\mathrm{L}$, et al. Prolonged presence of SARS-CoV-2 viral RNA in faecal samples. Lancet Gastroenterol Hepatol. 2020; 5(5): 434-435. doi:10.1016/S2468-1253(20)30083-2

7. Symptoms of Coronavirus | CDC. Accessed September 16, 2020 https://www.cdc.gov/coronavirus/2019-ncov/symptoms-testing/ symptoms.html

8. Li Q Guan X, Wu P, et al. Early Transmission Dynamics in Wuhan, China, of Novel Coronavirus-Infected Pneumonia. N Engl J Med. 2020 382(13): 1199-1207. doi:10.1056/NEJMoa2001316

9. Miller A, Reandelar MJ, Fasciglione K, et al. Correlation between universal BCG vaccination policy and reduced morbidity and mortality for COVID-19: an epidemiological study. medRxiv. Published online 2020. doi:https://doi.org/10.1101/2020.03.24.20042937
10. St Petersburg University scientists: vaccination against tuberculosis can reduce the spread of COVID-19 and ease its course - Saint Petersburg University. Accessed December 9, 2020. https://english. spbu.ru/news/4081-st-petersburg-university-scientists-vaccinationagainst-tuberculosis-can-reduce-the-spread-of-covid-19-and-easeits-course

11. Moorlag SJCFM, Arts RJW, van Crevel R, et al. Non-specific effects of BCG vaccine on viral infections. Clin Microbiol Infect. 2019; 25(12): 1473-1478. doi:10.1016/j.cmi.2019.04.020

12. Butkeviciute E, Jones CE, Smith SG. Heterologous effects of infant BCG vaccination: Potential mechanisms of immunity. Future Microbiol. 2018; 13(10): 1193-1208. doi:10.2217/fmb-2018-0026

13. Arts RJW, Moorlag SJCFM, Novakovic B, et al. BCG Vaccination Protects against Experimental Viral Infection in Humans through the Induction of Cytokines Associated with Trained Immunity. Cell Host Microbe. 2018; 23(1): 89-100.e5. doi:10.1016/j. chom.2017.12.010

14. Leentjens J, Kox M, Stokman R, et al. BCG vaccination enhances the immunogenicity of subsequent influenza vaccination in healthy volunteers: A randomized, placebo-controlled pilot study. J Infect Dis. 2015; 212(12): 1930-1938. doi:10.1093/infdis/jiv332

15. Aaby P, Roth A, Ravn H, et al. Randomized trial of BCG vaccination at birth to low-birth-weight children: Beneficial nonspecific effects in the neonatal period? J Infect Dis. 2011; 204(2): 245-252. doi:10.1093/ infdis/jir240

16. Garly ML, Martins CL, Balé $C$, et al. BCG scar and positive tuberculin reaction associated with reduced child mortality in West Africa: A non-specific beneficial effect of BCG? Vaccine. 2003; 21(21-22): 27822790. doi:10.1016/S0264-410X(03)00181-6

17. GHO | By category | BCG - Immunization coverage estimates by country. Accessed September 17, 2020. https://apps.who.int/gho/ data/node.main.A830?lang=en

18. BCG Wold Atlas. Accessed September 17, 2020. http://www.bcgatlas. org/index.php

19. Coronavirus Pandemic (COVID-19) - Statistics and Research - Our World in Data. Accessed September 17, 2020. https://ourworldindata. org/coronavirus

20. MAHLER HT, ALI PM. Review of Mass B . C . G . Project in India. Indian J Tuberc. 1954; (2): 108-116.

21. About us :: Central TB Division. Accessed September 24, 2020. https:// tbcindia.gov.in/index4.php?lang=1\&level=0\&linkid=399\&lid=2768

22. Lahariya C. A brief history of vaccines \& vaccination in India. Indian J Med Res. 2014; 139(April): 491-511.

23. Benévolo-de-Andrade TC, Monteiro-Maia R, Cosgrove $\mathrm{C}$, et al. BCG Moreau Rio de Janeiro: an oral vaccine against tuberculosis - review. Mem Inst Oswaldo Cruz. 2005; 100(5): 459-465. doi:10.1590/S007402762005000500002

24. Goodridge HS, Ahmed SS, Curtis N, et al. Harnessing the beneficial heterologous effects of vaccination. Nat Rev Immunol. 2016; 16(6): 392-400. doi:10.1038/nri.2016.43

25. Hamiel U, Kozer E, Youngster I. SARS-CoV-2 Rates in BCG-Vaccinated and Unvaccinated Young Adults. JAMA - J Am Med Assoc. 2020; 323(22): 2340-2341. doi:10.1001/jama.2020.8189

26. Meena J, Yadav A, Kumar J. BCG Vaccination Policy and Protection Against COVID-19. Indian J Pediatr. 2020; (June): 19-20. doi:10.1007/ s12098-020-03371-3

27. Hensel J, McAndrews KM, McGrail DJ, et al. Protection against SARSCoV-2 by BCG vaccination is not supported by epidemiological analyses. Sci Rep. 2020; 10(1): 1-9. doi:10.1038/s41598-02075491-x 
28. J ONeill LA, Netea MG. BCG-induced trained immunity: can it offer protection against COVID-19? Nat Rev Immunol. doi:10.1038/ s41577-020-0337-y
29. Bacille Calmette-Guérin (BCG) vaccination and COVID-19. Accessed September 18, 2020. https://www.who.int/news-room/ commentaries/detail/bacille-calmette-guérin-(bcg)-vaccinationand-covid-19 